\title{
A radio continuum study of the Magellanic Clouds
}

\section{Catalogues of radio sources in the Small Magellanic Cloud at 1.42, $2.45,4.75,4.85$ and $8.55 \mathrm{GHz}^{\star}$}

\author{
M.D. Filipovićc ${ }^{1,2}$, P.A. Jones ${ }^{1}$, G.L. White ${ }^{1}$, R.F. Haynes ${ }^{2,1}$, U. Klein ${ }^{3}$, and R. Wielebinski ${ }^{4}$ \\ 1 University of Western Sydney Nepean, P.O. Box 10, Kingswood, NSW 2747, Australia \\ fica@st.nepean.uws.edu.au; g.white@st.nepean.uws.edu.au; p.jones@st.nepean.uws.edu.au \\ 2 Australia Telescope National Facility, CSIRO, P.O. Box 76, Epping, NSW 2121, Australia \\ mfilipovic@atnf.csiro.au; rhaynes@atnf.csiro.au \\ 3 Radioastronomisches Institut der Universitat Bonn, Auf dem Hügel 71, D-53121 Bonn, Germany \\ uklein@astro.uni-bonn.de \\ 4 Max-Planck-Institut für Radioastronomie, Auf dem Hügel 69, D-53121 Bonn, Germany \\ p647gbr@mpifr-bonn.mpg.de
}

Received May 6; accepted May 7, 1996

\begin{abstract}
We present catalogues of radio sources in the Small Magellanic Cloud from observations with the Parkes radio telescope at $1.42,2.45,4.75$ and $8.55 \mathrm{GHz}$, and an additional catalogue from the Parkes-MIT-NRAO survey at $4.85 \mathrm{GHz}$. A total of 224 sources were detected at at least one of these frequencies, 60 of which are reported here for the first time as radio sources. We compare positions and flux densities of these sources with previously published results and find no significant positional displacement or flux discrepancies.
\end{abstract}

Key words: Magellanic Clouds — radio continuum: galaxies; general - catalogue

\section{Introduction}

The sensitive radio surveys of the Magellanic Clouds (MCs) were undertaken at $1.42,2.45,4.75,8.55 \mathrm{GHz}$ between 1988 and 1991 using the Parkes 64-m radio telescope (Haynes et al. 1986, 1991). In addition to these specific surveys, a part of the Parkes-MIT-NRAO (PMN) all-sky survey at $4.85 \mathrm{GHz}$ by Wright et al. (1994) covered the field of the MCs.

The catalogues of the radio sources in the Large Magellanic Cloud (LMC) at these five frequencies were published in Filipović et al. (1995; hereafter Paper IV).

Send offprint requests to: M.D. Filipović

* Tables 2-7 are only available electronically at the CDS via ftp 130.79.128.5 or at

http://cdsweb.u-strasbg.fr/Abstract.html
An additional catalogue of the LMC sources at $2.30 \mathrm{GHz}$ appears in Filipović et al. (1996).

In this paper we analyse the five surveys of the Small Magellanic Cloud (SMC) region and catalogue the discrete radio sources seen at these observing frequencies. We address issues related to the calibration of the surveys: source positional errors and the flux density calibration.

The astrophysical interpretation of sources found in the surveys will be presented in later papers. In Paper VI of this series (Filipovic et al. 1997, in prep.), we shall compare these radio surveys with the ROSAT All-Sky surveys and in Papers VII and VIII we shall attempt to classify all MCs sources and look into their nature and distribution throughout the MCs.

\section{The five Parkes surveys}

Table 1 lists the characteristics of the five SMC surveys used in this study of discrete sources. We consider the region of $\sim 6^{\circ} \times 7^{\circ}$ centred on Right Ascension (B1950) $00^{\mathrm{h}} 55^{\mathrm{m}} 00^{\mathrm{s}}$ and Declination (B1950) $-73^{\circ} 00^{\prime} 00^{\prime \prime}$ which cover $\sim 42$ square degrees between RA (B1950) $=00^{\mathrm{h}} 12^{\mathrm{m}}$ to $01^{\mathrm{h}} 38^{\mathrm{m}}$ and Dec $(\mathrm{B} 1950)=-69^{\circ} 40^{\prime}$ to $-76^{\circ} 20^{\prime}$.

The four primary surveys of the SMC at 1.42, 2.45, 4.75 and $8.55 \mathrm{GHz}$ were made between 1988 and 1991 using the Parkes 64-m telescope fitted with the Australia Telescope National Facility's prototype receiver package (Haynes et al. 1986, 1991). Observations at these four frequencies were made by scanning the telescope across the SMC in right ascension and declination at driving rates in the range $1^{\circ}$ to $4^{\circ} \mathrm{min}^{-1}$. At each observing frequency we used 1-s integration for each datum point and made 
Table 1. Parameters of the five radio surveys of the SMC

\begin{tabular}{ccccc}
\hline \hline $\begin{array}{c}\text { Frequency } \\
(\mathrm{GHz})\end{array}$ & $\begin{array}{c}\text { Beam } \\
\text { Size } \\
\text { (arcmin) }\end{array}$ & $\begin{array}{c}\text { Field } \\
\text { Size } \\
(\text { Degree })\end{array}$ & $\begin{array}{c}\text { rms } \\
\text { Noise } \\
\left(\mathrm{mJy} \mathrm{beam}^{-1}\right)\end{array}$ & $\begin{array}{c}\text { No. of } \\
\text { Sources } \\
\text { Detected }\end{array}$ \\
\hline 1.42 & 13.8 & $7.5^{\circ} \times 7.5^{\circ}$ & 15 & 85 \\
2.45 & 9 & $7^{\circ} \times 7^{\circ}$ & 10 & 107 \\
4.75 & 4.5 & $6.5^{\circ} \times 4.5^{\circ}$ & 6 & 99 \\
4.85 & 4.9 & $7.5^{\circ} \times 7.5^{\circ}$ & 5 & 187 \\
8.55 & 2.7 & $4^{\circ} \times 3^{\circ}$ & 10 & 41 \\
\hline
\end{tabular}

2.3 measurements across each beam size in the scanning direction. The beam size ranged from $13.8^{\prime}$ to $2.7^{\prime}$ depending on the observing frequency (Table 1). The resulting images exhibited some scanning "noise" (caused by variations in the absorbing emission characteristics of the Earth's atmosphere) which depended on the scanning direction. These scanning effects have been efficiently removed by the so-called "basket-weaving" technique developed at the MPIfR (Sieber et al. 1979).

Calibration was undertaken by frequently observing sources with known integrated flux density and position, and by mapping them in the same manner as for the MCs (Haynes et al. 1991; Paper IV). The main source for fluxdensity calibration was Hydra A. The adopted integrated flux density scale is that determined by Baars et al. (1977). As secondary calibrators (mainly used for pointing calibration) we chose the sources 3C 348, 3C 353, B0332-403, B0843-336, B1819-67, B1934-638 and B2356-61.

For the $1.42-\mathrm{GHz}$ survey, the primary calibrator was Virgo A (for which an integrated flux density of $213 \mathrm{Jy}$ was assumed), while the secondary calibrators were Hydra A, 3C 138, B1414+11, B1814-63 and B1819-67 (Haynes et al. 1986).

Our fifth study at $4.85 \mathrm{GHz}$ used a public-release FITS image of the SMC obtained from the PMN survey. The PMN Southern Survey was made with the Parkes telescope in 1992 (Griffith et al. 1991, 1994; Griffith \& Wright 1993; Wright et al. 1994). Radio images between declination range $-88^{\circ}<$ Dec $<-37^{\circ}$ at $4.85 \mathrm{GHz}$ are available in FITS format with a $4.9^{\prime}$ initial resolution and an rms. noise between 5 and $13 \mathrm{mJy}_{\text {beam }}{ }^{-1}$. We have taken these PMN survey FITS images which cover the region of the SMC and used the NOD2 source-fitting package to determine source positions and flux densities.

\section{Source fitting}

All data reduction for this study used the NOD2 software package (Haslam 1974), modified for the Parkes telescope data. The automatic two-dimensional elliptical Gaussianfitting algorithms implemented in the NOD2 package were used to search for sources and to determine source positions, flux densities and extension. A planar base level was fitted to the region of every source detected in the survey and the position and flux intensity of the discrete sources were then determined. Sources were catalogued if their fitted Gaussian flux intensity was at least 5 times the rms. noise level $(5 \sigma)$ in the image (Table 1) or seen in at least two different frequency images but not weaker than $4 \sigma$. The validity of each source was then checked by inspecting the images and, for those regions in which there were clearly more than one discrete source, multi-source Gaussian fitting was used to model the extended emitting region.

\section{Radio source catalogue}

\subsection{The tables}

Tables 2 to 6 are the catalogues of discrete radio sources found in the SMC surveys ${ }^{1}$. In these tables, we used the same format as for the paper on the LMC (Paper IV) i.e. the source name (Col. 1) is derived from the standard IAU system of source nomenclature (Dickel et al. 1987; Lortet et al. 1994), namely - SMC Bhhmm-ddmm (e.g., SMC B0054-7254). The letter "B" in Col. 1 indicates that the source positions (Cols. 2 and 3) are for equinox and equator B1950. For easier cross-checking we have adopted the reference source name based on the position at the highest frequency, since that position will be the most accurate. In Col. 4 the symbol (e) is used to flag sources that are significantly extended. The definition of extension is discussed below in Sect. 4.2. The integrated flux densities are listed in Col. 5.

Table 7 gives the cross-references for all sources in Tables 2 to 6. Column 3 (Other Names and Comments) uses the following abbreviations:

$\begin{array}{ll}N & \text { Henize (1956) (e.g., N 66), } \\ S & \text { McGee et al. (1976) (e.g., S 17), } \\ P K S & \text { Otrupcek \& Wright (1991) } \\ & \text { (e.g. PKS B0057-724), } \\ M C 4 & \text { Clarke et al. (1976) (e.g., MC4 B0057-724), } \\ M R C & \text { Large et al. (1981) (e.g., MRC B0027-698), } \\ M A & \text { Mills \& Aller (1971) (e.g., MA B0103-723), } \\ P M N & \text { Wright et al. (1994) (e.g., PMN J0059-7210), } \\ N G C & \text { Sinnott (1988) (e.g., NGC 346), } \\ D E M S & \text { Davies et al. (1976) (e.g., DEMS 103), } \\ 1 E & \text { Seward \& Mitchell (1981) } \\ & \text { (e.g., 1E 0057.6-7228), } \\ I K T & \text { Inoue et al. (1983) (e.g., IKT 18), } \\ L I-S M C & \text { Israel et al. (1993) (e.g., LI-SMC 131), } \\ W W & \text { Wang \& Wu (1992) (e.g., WW 44) and } \\ B K G S & \text { Bruhweiler et al. (1987) (e.g. BKGS 1A) }\end{array}$

1 These catalogues are now available in machine-readable form and can be obtained on request from the authors via Internet:mfilipovic@atnf.csiro.au or fica@st.nepean.uws.edu.au 


\subsection{Source extension}

The basket-weaving analysis, gridding and scanning process used during the observations slightly broadens the full width half maximum (FWHM) beam size. We have determined the effective beam size from the distribution of the fitted widths after excluding the tail of very extended sources. We excluded sources greater than 5 times the median size and then recalculated the median until the process converged ${ }^{2}$. The definition adopted here for a significantly extended source is that the fitted size (solid angle) of the source is twice that of the effective beam size. The true angular size of the sources is the observed size deconvolved with the effective beam size. Adopting this definition, between $5 \%$ and $20 \%$ (depending on the observing frequency) of all our sources in the SMC field are extended. For details about nominal beam size, effective beam size, uncertainty in the effective beam size and the number of extended sources, see Table 8 .

Table 2. Nominal and effective beam sizes, standard deviation of effective source sizes and number of sources considered as clearly extended

\begin{tabular}{ccccc}
\hline \hline $\begin{array}{c}\text { Frequency } \\
(\mathrm{GHz})\end{array}$ & $\begin{array}{c}\text { Nominal } \\
\text { Beam Size } \\
\text { (arcmin) }\end{array}$ & $\begin{array}{c}\text { Effective } \\
\text { Beam Size } \\
\text { (arcmin) }\end{array}$ & $\begin{array}{c}\text { Standard } \\
\text { Deviation } \\
\text { (arcmin) }\end{array}$ & $\begin{array}{c}\text { No. of } \\
\text { Extended } \\
\text { Sources }\end{array}$ \\
\hline 1.42 & 13.8 & 16.8 & 1.9 & 9 \\
2.45 & 9 & 10.1 & 1.1 & 12 \\
4.75 & 4.5 & 5.3 & 0.7 & 11 \\
4.85 & 4.9 & 5.2 & 0.6 & 12 \\
8.55 & 2.7 & 3.6 & 0.7 & 12 \\
\hline
\end{tabular}

\section{Calibration procedures}

\subsection{Position calibration and uncertainties in position}

The accuracy of the positions for all sources in our catalogues is limited by the pointing accuracy and beam size of the Parkes telescope. We have compared the positions of 20 radio sources from the MC4 catalogue (Clarke et al. 1976) with positions from our 8.55-GHz catalogue (Table 6 ) and have found no significant positional bias between our radio and MC4 catalogue positions $\left[\Delta \mathrm{RA}=-4^{\prime \prime} \pm 6^{\prime \prime}\right.$, $\left.\Delta \mathrm{Dec}=3^{\prime \prime} \pm 5^{\prime \prime}(8.55 \mathrm{GHz}-\mathrm{MC} 4)\right]$.

We have also compared our $4.85-\mathrm{GHz}$ catalogue positions (Table 5) with the published PMN survey source positions (Wright et al. 1994) for 146 common objects. Again, the positional difference is satisfactory $[\Delta \mathrm{RA}=$ $\left.2^{\prime \prime} \pm 2^{\prime \prime}, \Delta \mathrm{Dec}=2^{\prime \prime} \pm 2^{\prime \prime}(4.85 \mathrm{GHz}-\mathrm{PMN})\right]$ with standard deviations in the differences of $28^{\prime \prime}$ and $21^{\prime \prime}$, respectively.

\footnotetext{
2 The median rather then mean was used since the distribution was not Gaussian.
}

An additional comparison between accurate radio positions $\left(<0.5^{\prime \prime}\right)$ for three widely separated sources, B0020-747, B0045-757 and B0100-760, in both White et al. (1991) and our catalogues, also shows no significant positional bias.

The accuracy of source positions depends on the observing frequency and the source extension. For example, by comparing the positions of sources at the different radio frequencies, we conclude that the errors in position have a standard deviation of $16^{\prime \prime} \pm 3^{\prime \prime}$ in both right ascension and declination at $8.55 \mathrm{GHz}$. Positional accuracies of individual sources at each frequency are presented in Table 9 and are similar to those for the LMC (Paper IV). For each source, these were determined by comparing the 8.55$\mathrm{GHz}$ position with the position determined at the other frequencies. These positional accuracies represent $13 \pm 3 \%$ of the Parkes telescope beam size.

Table 3. Positional and flux density uncertainties for the SMC surveys. Uncertainties in flux density $\left(\Delta S=\sqrt{A^{2}+(B \times S)^{2}}\right)$

\begin{tabular}{ccccc}
\hline \hline $\begin{array}{c}\text { Frequency } \\
(\mathrm{GHz})\end{array}$ & $\begin{array}{c}\Delta \mathrm{RA} \\
(\operatorname{arcsec})\end{array}$ & $\begin{array}{c}\Delta \mathrm{Dec} \\
(\operatorname{arcsec})\end{array}$ & $\begin{array}{c}A \\
(\mathrm{Jy})\end{array}$ & $\begin{array}{c}B \\
(\%)\end{array}$ \\
\hline 1.42 & 109 & 111 & 0.017 & 14.9 \\
2.45 & 72 & 65 & 0.029 & 11.8 \\
4.75 & 28 & 25 & 0.003 & 7.8 \\
4.85 & 20 & 24 & 0.015 & 6.2 \\
8.55 & 16 & 16 & 0.030 & 11.1 \\
\hline
\end{tabular}

\subsection{Flux density calibration and uncertainties in the flux densities}

\subsubsection{Comparison with the Parkes (PKSCAT-90) Catalogue}

To establish the relative flux density scales of our catalogues at $1.42,2.45,4.75$ and $8.55 \mathrm{GHz}$ we have compared our flux density with those from the PKSCAT-90 catalogue (Otrupcek \& Wright 1991) which includes sources from surveys at $1.40 \mathrm{GHz}$ (Mathewson \& Healey 1964; McGee et al. 1976), 2.70 GHz (McGee et al. 1976; Bolton \& Butler 1975; Broten 1972), $5.00 \mathrm{GHz}$ and $8.80 \mathrm{GHz}$ (McGee et al. 1976).

Few sources in the SMC are contained in the PKSCAT90 catalogue at $1.40 \mathrm{GHz}$. Flux densities at this frequency have been estimated (McGee et al. 1976) for a few strong sources from the 21-cm contour map of Mathewson \& Healey (1964). Figure 1a shows a comparison of source flux densities at $1.42 \mathrm{GHz}$ from our survey with the estimated 1.40-GHz flux densities from PKSCAT-90 and McGee et al. (1976). The median of the ratio between our estimates and PKSCAT-90 is 1.01 (our flux densities are higher) with a standard deviation of 0.30 . 

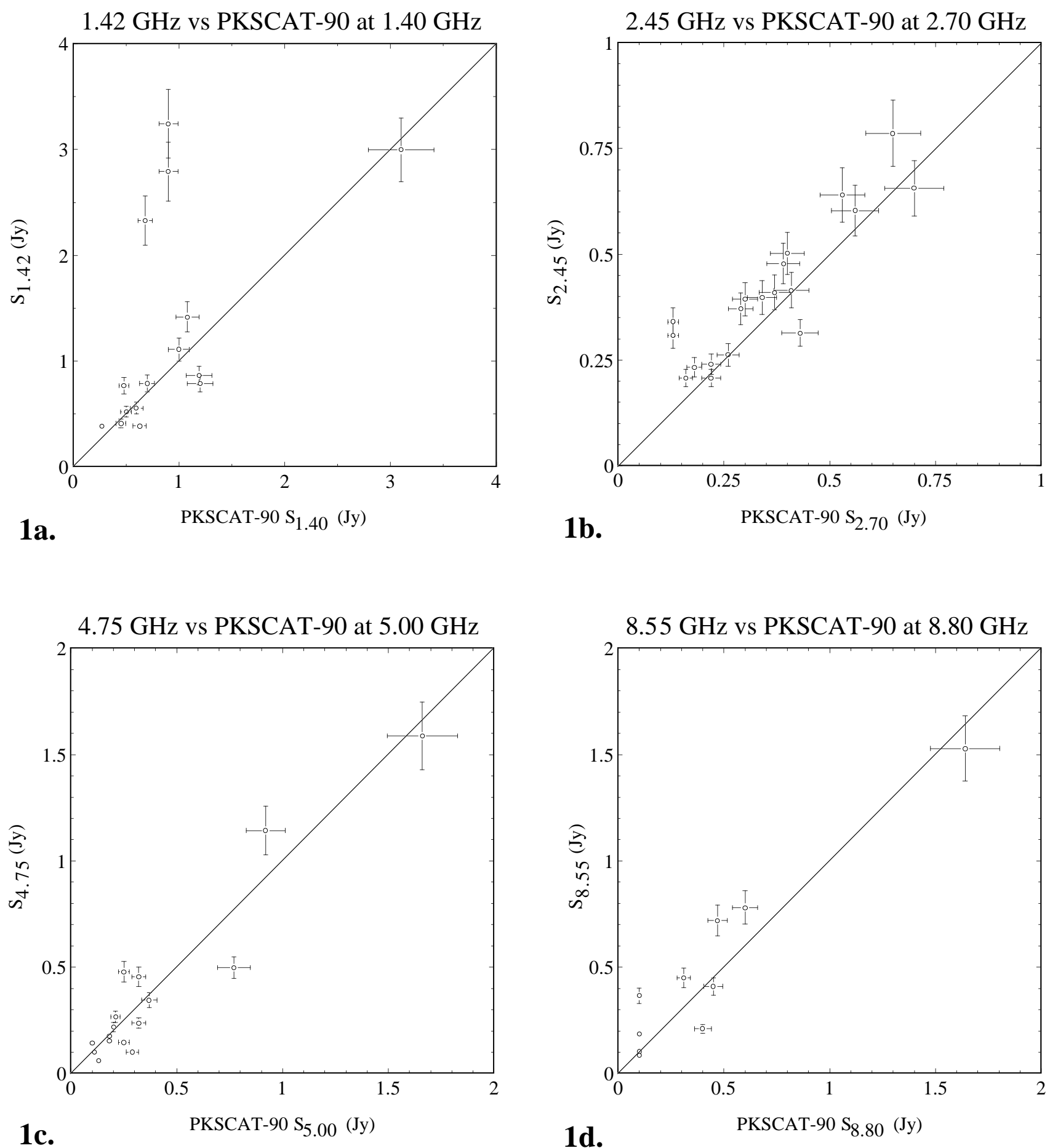

Fig. 1. Comparisons of flux densities in Tables 2-6 with flux densities from the PKSCAT-90 source compilation and that of McGee et al. (1976) at similar frequencies. a) gives flux densities from Table 2 (measured at $1.42 \mathrm{GHz}$ ) compared with the 1.40-GHz flux estimates from PKSCAT-90 and McGee et al. (1976). Similarly, b), c), d) compare flux densities in Tables 3 $(2.45 \mathrm{GHz}), 4(4.75 \mathrm{GHz})$ and $6(8.55 \mathrm{GHz})$ with PKSCAT-90 fluxes at 2.70, 5.00 and $8.80 \mathrm{GHz}$, respectively. Details of the comparisons are given in Sect. 5.2.1 
Figure $1 \mathrm{~b}$ presents a comparison of the integrated flux density from our survey at $2.45 \mathrm{GHz}$ versus the flux density at $2.70 \mathrm{GHz}$ (McGee et al. 1976; Bolton \& Butler 1975; Broten 1972) for 18 sources in common (excluding four very extended sources). The resulting median is 1.14 with a standard deviation of 0.16 .

Figure 1c shows a comparison of the integrated flux density of sources at $4.75 \mathrm{GHz}$ versus flux density at $5.00 \mathrm{GHz}$ from McGee et al. (1976). From our new survey at $4.75 \mathrm{GHz}$, we found 15 of the 27 sources. A comparison of 15 of these sources observed by McGee et al. (1976) and by us shows reasonably good agreement between the flux densities. The median ratio is 0.95 with a standard deviation of 0.40 .

The comparison of flux density at $8.55 \mathrm{GHz}$ versus flux density at $8.80 \mathrm{GHz}$ (McGee et al. 1976) given in Fig. 1d, shows a median ratio of 1.04 with a standard deviation of 0.41 . Only nine sources are in common.

From all of these comparisons, shown in Figs. 1a to 1d, only a very few sources have spectra that do not conform to a power-law relationship. Such sources are often extended and blended with other surrounding sources or are variable. In summary, the flux densities of our catalogues (Tables 2 to 6 ) agree well with those of the catalogues used for comparison.

\subsubsection{Comparison with the Southern Hemisphere PMN survey}

In Fig. 2a we compare our fitted flux densities at $4.85 \mathrm{GHz}$ (Table 5) with flux densities as published in the PMN source catalogue (Wright et al. 1994) at $4.85 \mathrm{GHz}$ for 148 sources in common. The flux densities agree very well (ratio $=1.04, \mathrm{SD}=0.33$ ) except for a very few clearly extended sources where the source-fitting algorithm used for the PMN catalogue is not optimized. This problem has been addressed in Paper IV and in Wright et al. (1994).

Source flux densities determined from the PMN FITS images, using the NOD2 package, also agree well with the fitted flux densities from the 4.75-GHz survey (Fig. 2b), indicating an excellent consistency. The ratio $\left(S_{4.85} \mathrm{GHz} \backslash\right.$ $\left.S_{4.75 \mathrm{GHz}}\right)$ is 0.99 with a standard deviation of 0.25 .

\subsubsection{Uncertainties in flux densities}

Following Paper IV, we have compared the flux densities at our five frequencies with interpolated flux densities at the same frequencies from PKSCAT-90 (Otrupcek \& Wright 1991). This type of analysis gives the uncertainty in flux density in the form:

$$
\Delta S=\sqrt{A^{2}+(B \times S)^{2}}
$$

where $A$ and $B$ are constants. The flux-densityindependent component of uncertainty $(A)$ has been computed from the rms differences for weak sources (where flux-density-dependent uncertainties are negligible) and

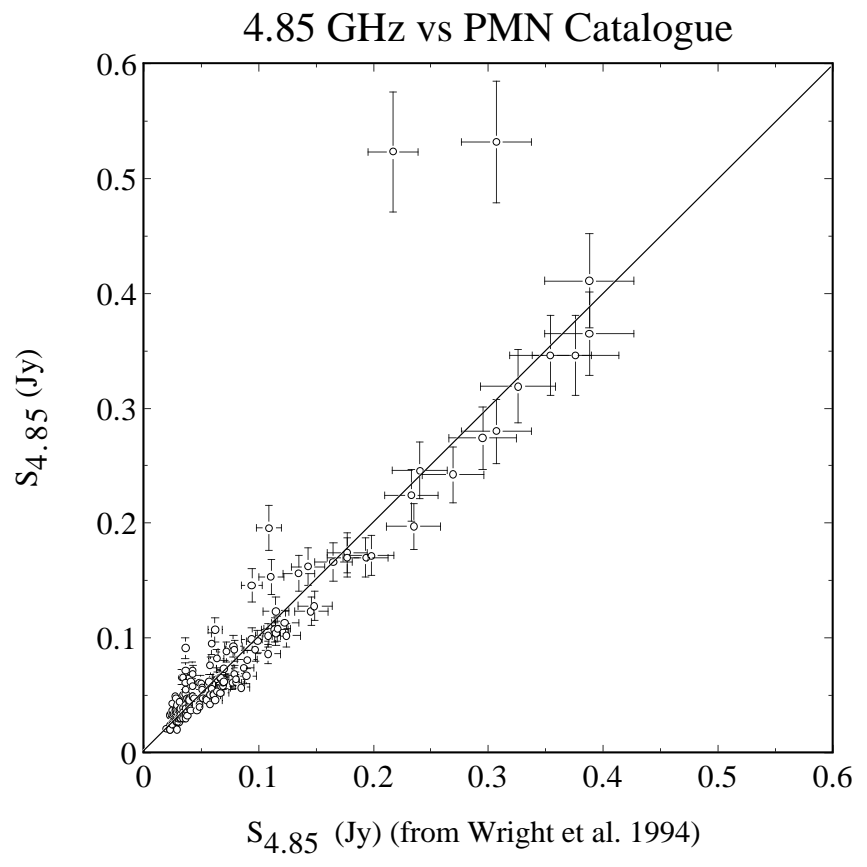

2a.

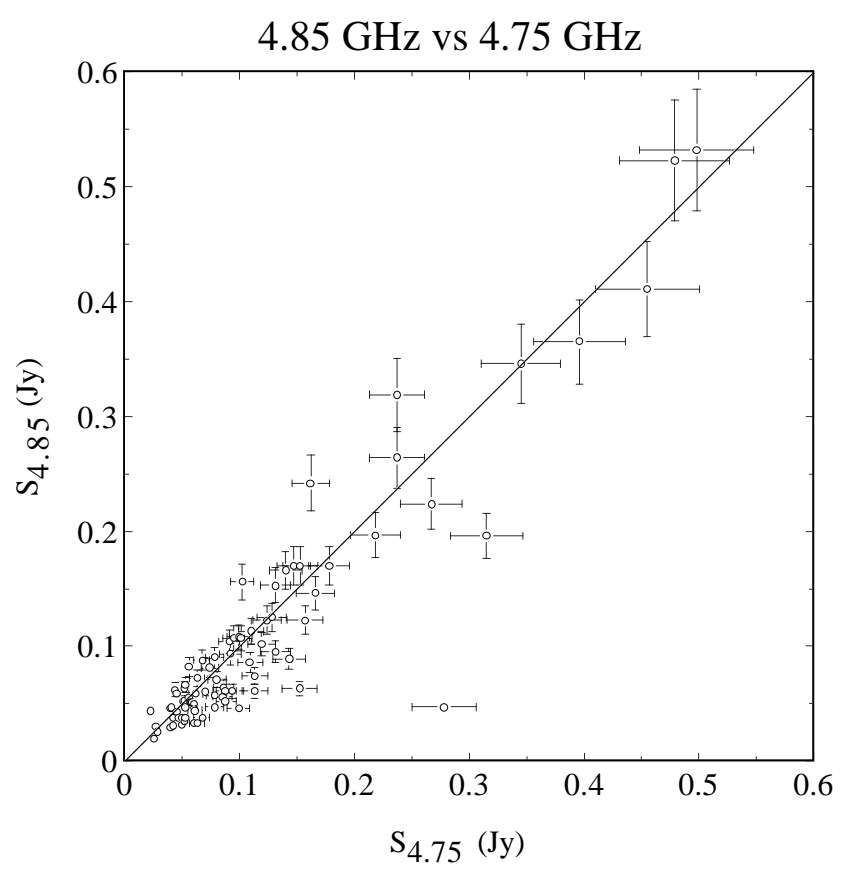

$2 b$.

Fig. 2. a) compares $4.85-\mathrm{GHz}$ flux densities (from Table 5) with published flux densities determined by Wright et al. (1994) from the 4.85-GHz Parkes-MIT-NRAO (PMN) survey. b) compares the $4.85-\mathrm{GHz}$ (Table 5) with the $4.75-\mathrm{GHz}$ flux densities (Table 4). Details of these comparisons are given in Sect. 5.2.2 
the flux-density-dependent uncertainties $(B)$ have been computed from the rms differences for strong sources. Assuming an equal rms noise for both our surveys and the PKSCAT-90 compilation, we obtain the uncertainties listed in Table 9 which are similar to those for the LMC (Paper IV).

For all but the very weakest sources, the uncertainty in flux density is dominated by the flux-density-dependent component which is $\sim 10 \pm 3 \%$.

\section{Conclusions}

This work presents new catalogues of radio sources in the SMC at five radio frequencies: $1.42 \mathrm{GHz}$ (86 sources), $2.45 \mathrm{GHz}$ (107 sources), $4.75 \mathrm{GHz}$ (99 sources), $4.85 \mathrm{GHz}$ (187 sources) and $8.55 \mathrm{GHz}$ (41 sources). There is a total of 224 radio sources, 60 of which are new. We have already identified some of these new sources at other wavelengths. Further analysis of the SMC discrete sources will appear in following papers, discussing the radio spectral indices and comparisons with X-ray, infrared and optical data.

Acknowledgements. We are very grateful to R. Duncan, P. Müller and C. Sheerman for help with the NOD2 package and to D.E. Goddard and L. Staveley-Smith for comments on the manuscript. M.D.F. acknowledges an Australian Government Overseas Postgraduate Research Scholarship and considerable support from the Australia Telescope National Facility (CSIRO) and the Max-Planck-Institut für Radioastronomie (MPIfRA). This work is also supported by an Australian Research Council (ARC) research grant.

\section{References}

Baars J.W.M., Genzel R., Pauliny-Toth I.I.K., Witzel A., 1977, A\&A 61, 99

Bolton J.G., Butler P.W., 1975, Aust. J. Phys. Astrophys. Suppl. 34, 33

Broten N.W., 1972, Aust. J. Phys. 25, 599

Bruhweiler F.C., Klinglesmith D.A., Gull T.R., Sofia S., 1987, ApJ 317, 152

Clarke J.N., Little A.G., Mills B.Y., 1976, Aust. J. Phys. Astrophys. Suppl. 40, 1
Davies R.D., Elliott K.H., Meaburn J., 1976, Mem. R. astr. Soc. 81,89

Dickel H.R., Lortet M.-C., de Boer K.S., 1987, A\&AS 68, 75

Filipović M.D., Haynes R.F., White G.L., Jones P.A., Klein U., Wielebinski R., 1995, A\&AS 111, 311 (Paper IV)

Filipović M.D., White G.L., Haynes R.F., Jones P.A., Klein U., Wielebinski R., 1996, A\&AS 120, 77

Griffith M., Wright A.E., Hunt A.J., et al., 1991, Proc. Astron. Soc. Aust. 9, 243

Griffith M., Wright A.E., 1993, AJ 105, 1666

Griffith M., Wright A.E., Burke B., Ekers R.D., 1994, ApJS 90, 179

Haslam C.G.T., 1974, A\&AS 15, 333

Haynes R.F., Klein U., Wielebinski R., Murray J.D., 1986, A\&A 159, 22

Haynes R.F., Klein U., Wayte S.R., et al., 1991, A\&A 252, 475

Henize K.G., 1956, ApJS 2, 315

Inoue H., Koyama K., Tanaka Y., 1983, in: Danziger I.J., Gorenstein P. (eds.) Proc. IAU Symp. 101, Supernova Remnants and their X-Ray Emission. Reidel, Dordrecht, p. 535

Israel F.P., Johansson L.E.B., Lequeux J., et al., 1993, A\&A 276,25

Large M.I., Mills B.Y., Little A.G., Crawford D.F., Sutton J.M., 1981, MNRAS 194, 693

Lortet M.-C., Borde S., Ochsenbein F., 1994, A\&AS 107, 193

Mathewson D.S., Healey J.R., 1964, in: Kerr F.J., Rodgers A.W. (eds.) The Galaxy and the Magellanic Clouds. Canberra, Aust. Acad. Sci., p. 283

McGee R.X., Newton L.M., Butler P.W., 1976, Aust. J. Phys. 29, 329

Mills B.Y, Aller L.H., 1971, Aust. J. Phys. 24, 609

Otrupcek R.E., Wright A.E., 1991, Proc. Astron. Soc. Aust. 9, 170

Seward F.D., Mitchell M., 1981, ApJ 243, 736

Sieber W., Haslam C.G.T., Salter C.J., 1979, A\&A 74, 361

Sinnott R.W., NGC 2000, 1988. Cambridge Univ. Press, p. 46

Wang Q., Wu X., 1992, ApJS 78, 391

White G.L., Bunton J.D., Anderson M.W.B., et al., 1991, MNRAS 248, 398

Wright A.E., Griffith M., Burke B., Ekers R.D., 1994, ApJS 91, 111 